\title{
Mycobacteriophage-drived diversification of Mycobacterium abscessus
}

\author{
Mohamed Sassi ${ }^{1}$, Philippe Gouret ${ }^{2}$, Olivier Chabrol $^{2}$, Pierre Pontarotti ${ }^{2}$ and Michel Drancourt ${ }^{*}$
}

\begin{abstract}
Background: Mycobacterium abscessus is an emerging opportunistic pathogen which diversity was acknowledged by the recent description of two subspecies accommodating M. abscessus, Mycobacterium bolletii and Mycobacterium massiliense isolates.

Results: Here, genome analysis found 1-8 prophage regions in 47/48 M. abscessus genomes ranging from small prophage-like elements to complete prophages. A total of 20,304 viral and phage proteins clustered into 853 orthologous groups. Phylogenomic and phylogenetic analyses based on prophage region homology found three main clusters corresponding to M. abscessus, M. bolletii and M. massiliense. Analysing 135 annotated Tape Measure Proteins found thirteen clusters and four singletons, suggesting that at least 17 mycobacteriophages had infected M. abscessus during its evolution. The evolutionary history of phages differed from that of their mycobacterial hosts. In particular, 33 phage-related proteins have been horizontally transferred within M. abscessus genomes. They comprise of an integrase, specific mycobacteriophage proteins, hypothetical proteins and DNA replication and metabolism proteins. Gene exchanges, loss and gains which occurred in M. abscessus genomes have been driven by several mycobacteriophages.
\end{abstract}

Conclusions: This analysis of phage-mycobacterium co-evolution suggests that mycobacteriophages are playing a key-role in the on-going diversification of M. abscessus.

Reviewers: This article was reviewed by Eric Bapteste, Patrick Forterre and Eugene Koonin.

Keywords: Mycobacterium abscessus, Mycobacterium bolletii, Mycobacterium massiliense, Prophages, Mycobacteriophages

\section{Background}

Mycobacterium abscessus is a non-tuberculous species comprising emerging opportunistic pathogens [1] responsible for sporadic cases and outbreaks of skin and softtissue infections following surgical and cosmetic practices [2-4]; catheter-related bacteremia [5,6]; and respiratory tract infections in patients with underlying lung disorders, particularly cystic fibrosis [7-13]. M. abscessus is broadly resistant to antibiotics and the cure of localized $M$. abscessus infection may require surgery [14].

Previous phenotypic [3] and genetic [15-17] analyses showed diversity among collections of $M$. abscessus isolates acknowledged by the description of two subspecies,

\footnotetext{
* Correspondence: michel.drancourt@univ-amu.fr

'Unité de Recherche sur les Maladies Infectieuses et Tropicales Emergentes UMR CNRS 6236 IRD198, IFR48, Institut Méditerranée Infection, Aix Marseille Université, Marseille, France

Full list of author information is available at the end of the article
}

M. abscessus subsp. abscessus and M. abscessus subsp. bolletii [18-20]. Later taxon accommodates mycobacteria previously refered as Mycobacterium bolletii [21] and Mycobacterium massiliense [22]. M. abscessus can therefore be viewed as a complex of at least three different organisms $M$. abscessus, $M$. bolletii and M. massiliense and this nomenclature will be retained in this paper.

There are a few data regarding mycobacteriophages in $M$. abscessus complex including a $81-\mathrm{kb}$ prophage in the reference $M$. abscessus genome [1]. Also, we recently resolved the electron microscopy $3 \mathrm{D}$ structure of a M. bolletii mycobacteriophage named Araucaria [23]. However, the repertoire of $M$. abscessus phages and their evolutionary history within this bacterium is unknown and no systematic exploration for prophages and mycobacteriophages has been performed among additionally available sequenced $M$. abscessus genomes, leaving 
undetermined whether these initial observations were unique to some particular isolates or were representative of the $M$. abscessus species.

Here, exploiting genome sequence available for 48 M. abscessus mycobacteria by original bio-informatic analyses, we explored the repertoire of $M$. abscessus mycobacteriophages to gain insights into their evolution history compared to that of M. abscessus hosts.

\section{Methods}

\section{Establishing the repertoire of $M$. abscessus phages}

The genomes of $48 \mathrm{M}$. abscessus mycobacteria available in June 2013 were downloaded from Genbank (Table 1, Additional file 1). As for 47 unfinished genomes, the contigs were reoriented based on the $M$. abscessus type strain genome (GenBank GCF_000069185.1) used as reference using MAUVE software [24]. The prophage regions were detected using PHAST software [25]. Protein sequences were predicted in all genomes using prodigal software [26] in order to normalize prediction. M. abscessus panproteome was annotated using BlastP search with a cutoff E-value $<0.001$, percentage similarity $>30 \%$ and an alignment length $>50$ amino-acids against a home-made database (including PHAST database, Mimivirus, Marseillevirus and additional mycobacteriophage proteins). We further analyzed the $M$. abscessus complex genomes for Clustered Regularly Interspaced Short Palindromic Repeats (CRISPRs) using CRISPRs finder program [27].

\section{Determining M. abscessus phage phylogenies M. abscessus mycobacteria tree}

M. abscessus genomes were aligned using Muscle aligner implemented in Mauve software [24]. Mauve alignment generated an identity matrix which the identity scores range between 0 and 1 , where 0 indicates that no identical homologous nucleotides were found, and 1 indicates that every homologous nucleotide was identical. This matrix was then used to construct $M$. abscessus split network using Neighbor-Net algorithm in the package SplitsTree4 [28].

\section{M. abscessus phage tree}

The annotated viral and phage proteins were classified using OrthoMCL software [29]. Only protein sequences $>50$-amino acid residues were considered for further analyses. Homologous sequences were selected using the all-against-all BlastP algorithm [30] with an E value of $<10^{-5}$. Then, clustering of the orthologous sequences was analyzed using the Markov Cluster algorithm [31]. The inflation index of 1.5 was used to regulate cluster tightness (granularity). The resulting orthologous groups were used to construct a whole-genome network using the Neighbor-Net algorithm based on a gene content matrix. The similarity between two species is defined as the number of phage genes in common divided by the total number of genes of the two species. [32,33]. Using this matrix, we constructed also a heatmap clusterization using R package [http://www.r-project.org/].

\section{Detecting gene transfer events}

The orthologous groups identified by OrthoMCL were submitted to PhyloPattern for the analysis and manipulation of phylogenetic trees (within the DAGOBAH framework) [34,35]. The M. abscessus tree was used as a reference to infer topologies in order to detect gene gain and lost as previously described [35]. The results were submitted to FIGENIX [36] for phylogenetic reconstruction within the DAGOBAH framework as previously described [35]. The output generated by FIGENIX was submitted to the multi-agent system DAGOBAH, in which horizontal gene transfer (HGT) events were detected using an in-house-built transfer filter called HGT agent, as previously described [35,36]. This filter uses PhyloPattern to annotate each internal duplication node of the tree with three tags, including the recipient species, the donor species and external species [34]. Then, it applies a special phyletic pattern and searches the gene tree to find recipient species that are closer to donor species than to other external species that would otherwise be placed between the recipient and donor species in the species tree. In other words, a "donor" subtree must contain only species of a specific group and not those from the "recipient" group and vice versa and there should be no common species between the donor and external groups. Using HGT agent, one can specify the name of the donor and recipient species according to their usage.

\section{Results}

\section{The repertoire of $M$. abscessus phages}

Among 48 analysed genomes of $M$. abscessus, we found that only $M$. abscessus M154 encodes no prophage regions whereas the other $47 \mathrm{M}$. abscessus mycobacteria genomes harbour one to eight prophage regions. A total of 171 predicted prophage regions could be separated into four types i) intact prophages encoding structural proteins, lysis proteins, integration proteins and proteins necessary for replication and recombination ii) questionable prophages iii) incomplete prophage regions iv) small prophage-like elements (Table 1, Additional file 1).

In order to estimate the number of phages infecting M. abscessus, a phylogenetic tree was constructed based on Tape Measure Proteins (TMP) (Figure 1). The TMP was selected because it is typically the longest gene in mycobacteriophage genomes and because regions within the TMP gene are conserved [37].We could annotate 135 TMPs which clustered into thirteen groups and four singletons using orthoMCL. The TMP-based phylogenetic 
Table $1 M$. abscessus genomes properties and prophage regions

\begin{tabular}{|c|c|c|c|c|}
\hline Group & Strain & Genome lenght $\mathrm{Mb}$ & Genome GC\% & $\mathrm{N}^{\circ}$ of prophage regions \\
\hline \multirow[t]{22}{*}{ M. abscessus } & M. abscessus.CIP104536T & 5.09 & 62.7 & 1 \\
\hline & M93 & 5.08 & 64.2 & 4 \\
\hline & M94 & 5.1 & 64.2 & 2 \\
\hline & 4S-0116-R 4S_0116_R & 4.84 & 64 & 1 \\
\hline & 4S-0116-S 4S_0116_S & 4.84 & 64 & 1 \\
\hline & 4S-0726-RA 4S_0726_RA & 4.84 & 64 & 1 \\
\hline & 4S-0206 M4S_0206 & 4.86 & 64 & 2 \\
\hline & 4S-0303 4S_0303 & 4.86 & 64 & 2 \\
\hline & 4S-0726-RA 4S_0726_RB & 4.86 & 64 & 1 \\
\hline & 3A-0930-R 3A_0930_R & 5.27 & 64 & 8 \\
\hline & 3A-0119-R 3A_0119_R & 5.28 & 63.8 & 7 \\
\hline & 3A-0810-R M3A_0810_R & 5.29 & 64 & 8 \\
\hline & 3A-0122-R 3A_0122_R & 5.23 & 63.9 & 5 \\
\hline & 3A-0122-S 3A_0122_S & 5.23 & 63.9 & 6 \\
\hline & 3A-0731 3A_0731 & 5.39 & 64 & 8 \\
\hline & 3A-0930-R 3A_0930_S & 5.25 & 64 & 8 \\
\hline & 6G-0728-S 6G_0728_S & 5.32 & 64.1 & 2 \\
\hline & 6G-0125-S 6G_0125_S & 5.33 & 64.1 & 2 \\
\hline & 6G-0728-R M6G_0728_R & 5.34 & 64.1 & 2 \\
\hline & 6G-1108 6G_1108 & 5.34 & 64.1 & 2 \\
\hline & 6G-0125-R 6G_0125_R & 5.14 & 64.1 & 2 \\
\hline & 6G-0212 M6G_0212 & 5.14 & 64.1 & 2 \\
\hline \multirow[t]{22}{*}{ M. massiliense } & M. massiliense $\mathrm{BD}$ & 5.2 & 64.2 & 3 \\
\hline & M172 & 5.2 & 64.2 & 6 \\
\hline & $M 47 J 26$ & 4.87 & 64.1 & 3 \\
\hline & M154 & 4.8 & 64.1 & 0 \\
\hline & M18 & 4.89 & 64.2 & 1 \\
\hline & 2B-0107 M2B_0107 & 4.81 & 64.2 & 2 \\
\hline & 2B-0307 M2B_0307 & 4.81 & 64.2 & 2 \\
\hline & 2B-0912-R 2B_0912_R & 4.81 & 64.2 & 2 \\
\hline & 2B-0912-S 2B_0912_S & 4.81 & 64.2 & 1 \\
\hline & 2B-0626 M2B_0626 & 4.81 & 64.2 & 2 \\
\hline & B-1231 M2B_1231 & 4.81 & 64.2 & 2 \\
\hline & 1S_51_0915 & 4.89 & 64.2 & 3 \\
\hline & 1S-152-0930 & 4.9 & 64.2 & 3 \\
\hline & 1S_152_0914 & 4.9 & 64.2 & 3 \\
\hline & 5S-1215 5S_1215 & 5.21 & 64.1 & 6 \\
\hline & 5S-0421 5S_0421 & 5.24 & 64.1 & 5 \\
\hline & 5S-1212 5S_1212 & 5.24 & 64.1 & 6 \\
\hline & 5S-0304 5S_0304 & 5.25 & 64.1 & 6 \\
\hline & 5S-0708 5S_0708 & 5.25 & 64.1 & 6 \\
\hline & 5S-0817 5S_0817 & 5.25 & 64.1 & 6 \\
\hline & 5S-0921 M5S_0921 & 5.25 & 64.1 & 6 \\
\hline & 5S-0422 5S_0422 & 5.32 & 64.1 & 6 \\
\hline
\end{tabular}


Table 1 M. abscessus genomes properties and prophage regions (Continued)

\begin{tabular}{|c|c|c|c|c|}
\hline & M159 & 4.94 & 64.2 & 1 \\
\hline \multirow{3}{*}{ M. bolletii } & M115 & 4.98 & 64.1 & 3 \\
\hline & M. bolletii $\mathrm{BD}^{\top}$ & 5.05 & 64.2 & 3 \\
\hline & M24 & 5.51 & 64.2 & 7 \\
\hline
\end{tabular}

tree was constructed using MEGA software. The tree suggested that at least 17 different mycobacteriophages had infected $M$. abscessus, $M$. bolletii and M. massiliense during their evolution.

Based on homology between prophage regions of $M$. abscessus genomes, the $M$. abscessus group could be separated into three clusters, $M$. abscessus, $M$. massiliense and $M$. bolletii (Figure 2). Few exceptions were observed: strains M139 and 1S_51_0915 showed prophage region homology with the $M$. abscessus cluster while $M$. bolletii M24 showed prophage region homology with the M. massiliense cluster (Figure 2). Also, a 12-kb small prophagelike element is conserved within the M. abscessus and M. massiliense clusters. Analyzing 242,067 proteins of all $48 \mathrm{M}$. abscessus proteomes found 20,304 (8.4\%) proteins homologous to viral or phage proteins represented in Figure 3. These 20,304 proteins yielded 853 groups of orthologous proteins. All the species are represented in 239 groups $(28.02 \%)$. Only three $M$. abscessus genomes have unique genes, two viral proteins in M. abscessus M94, four mycobacteriophage proteins in M. abscessus M159 and two viral proteins in M. abscessus M172. The annotation of the prophage found $44 \%$ proteins to be implicated in DNA replication and bacterial or/and phage metabolism, 37\% were annotated as bacteriophage proteins (including structural, integration and terminase) and $14 \%$ proteins had no functional annotation. Interestingly, 289 proteins were annotated as holin and 75 as lysin protein. Twenty-five genomes including $M$. abscessus, M. bolletii and $M$. massiliense type strains encode

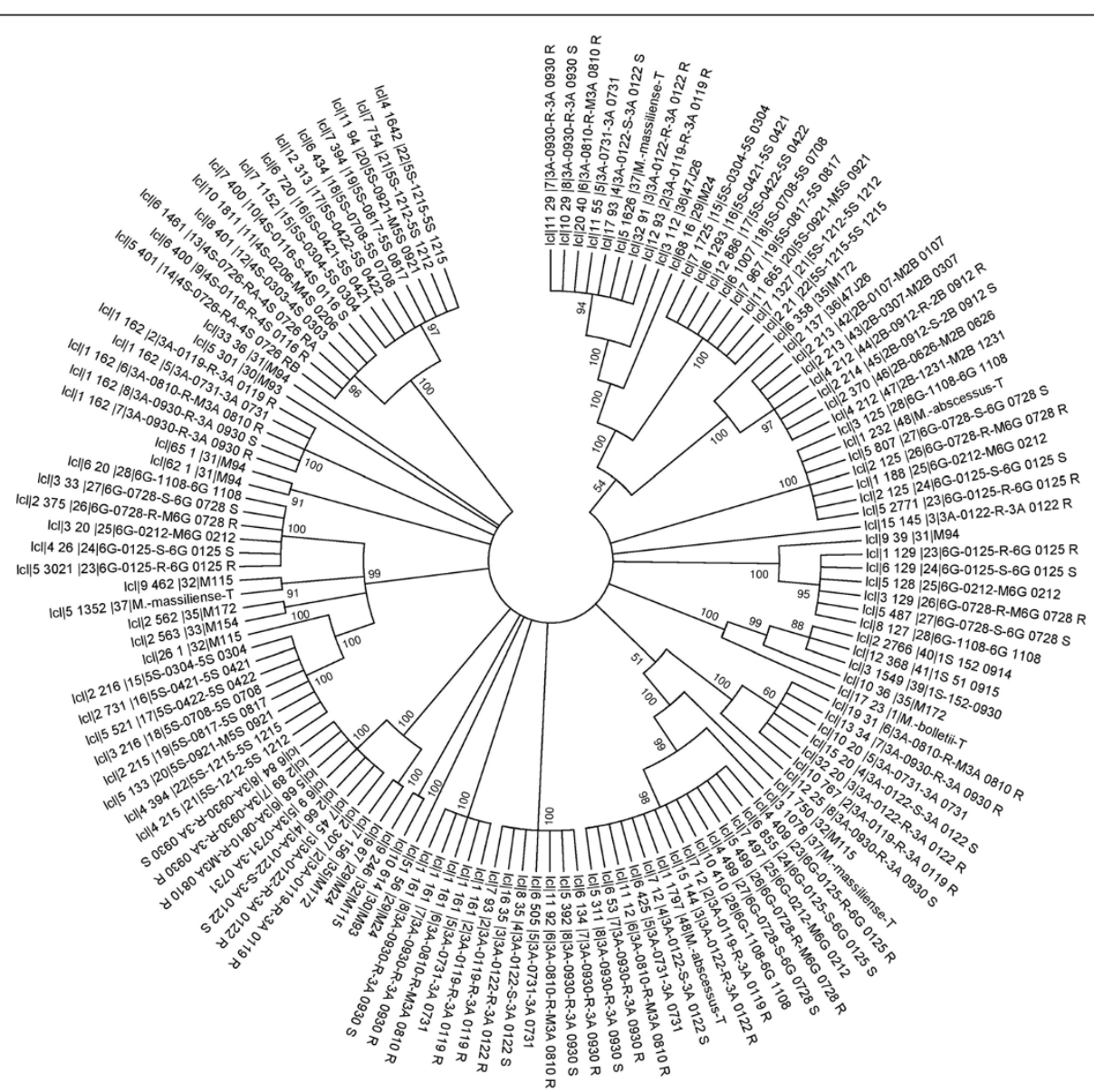

Figure 1 Phylogenetic tree based on annotated tape measure protein sequences using neighbour joining method. 


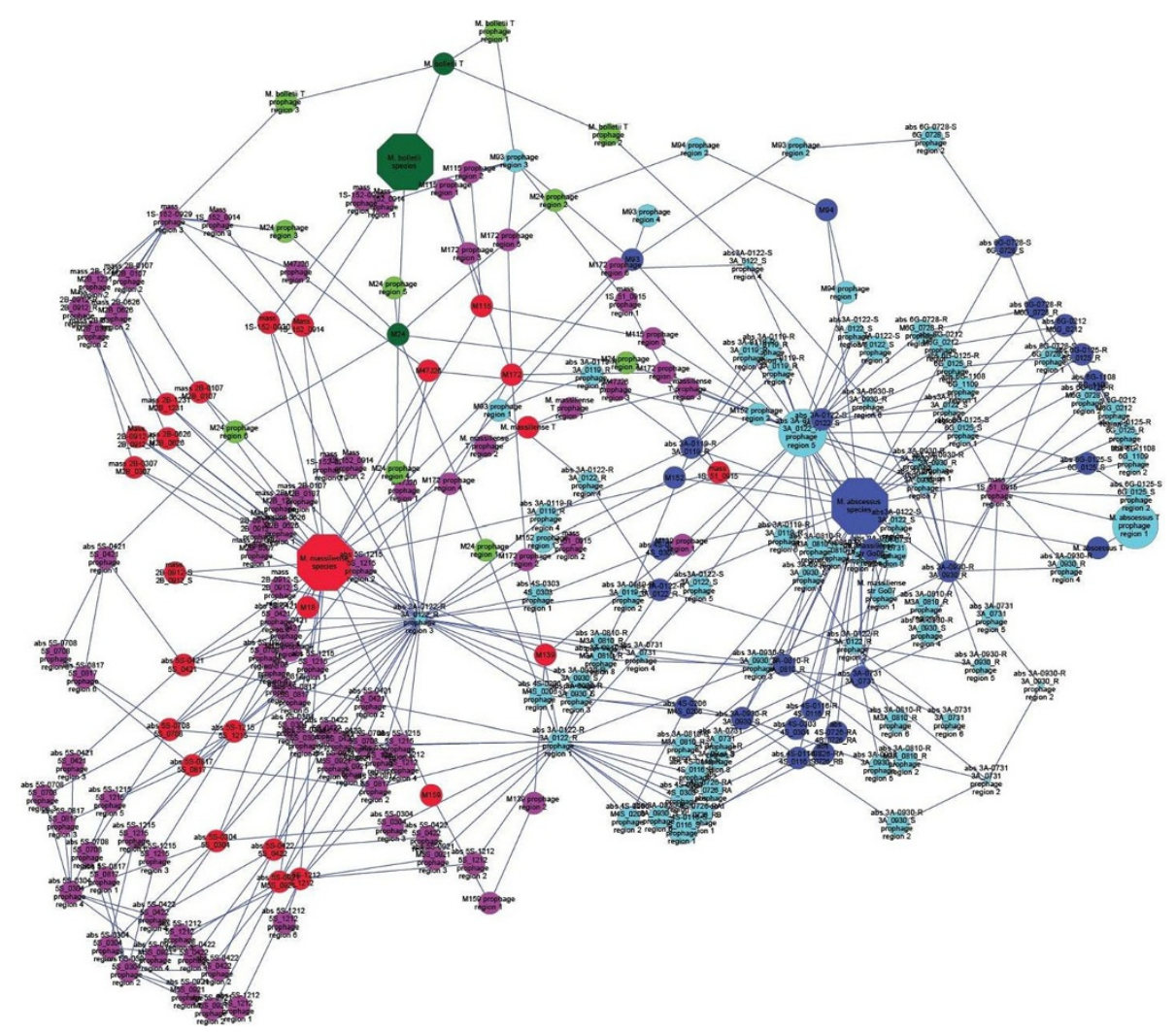

Figure 2 Interacting Map based on M. abscessus prophage region homology. Blue: M. abscessus species. Red: M. massiliense species. Green: M. bolletii species. The nodes represent the M. abscessus prophage regions. The node size correlates to the size of the M. abscessus prophage regions. The homologous regions were connected with edges.

endolysin-A and endolysin-B family lysin proteins. A total of $37.3 \%$ such proteins are homologous to lysin from mycobacteriophages, the other ones being homologous to lysin from phage infecting Firmicutes bacteria (Bacillus phages). Moreover, 156 proteins are repressor and antirepressor proteins of the lambda repressor $\mathrm{CI} / \mathrm{C} 2$ family (Lactobacillus phage and Staphylococcus phage), immunity repressor (Bacillus phage and Geobacillus phage) and Phage antirepressor protein KilAC domain (Rhodococcus phage). M. bolletii genome encodes only one $\mathrm{CI} / \mathrm{C} 2 \mathrm{re}-$ pressor homologous to the CI repressor from Bacillus phage and one putative repressor located out of the Araucaria genome. All other $M$. abscessus encode three to nine repressors. Only M. abscessus $6 \mathrm{G}$ and $M$. abscessus type strain encode antirepressors.

Twelve genomes including $M$. abscessus type strain encode no CRISPRs, seven genome including $M$. bolletii type strain encode one possible CRISPR, twelve genomes encode two possible CRISPRs, twelve genomes encode three possible CRISPRs and four genomes encodes four CRISPRs including M154, M115, M172 and M18 strains (Additional file 2).

\section{Phylogenomic and phylogenetic analyses M. abscessus tree}

The split network based on whole-genome content of $M$. abscessus shows the separation of $M$. abscessus strains into three main clusters respectively comprising M. abscessus, M. massiliense and M. bolletii genomes (Figure 4A). $M$. abscessus clusters comprise seven splits of a set of 22 strains. M. massiliense cluster comprises two sub-clusters; one sub-cluster forming the strains M159 and M115 and one sub-cluster forming 22 other strains. The $M$. massiliense cluster comprises 12 splits of a set of 24 strains. M. bolletii cluster comprises of two strains M. bolletii type strain and strain M24.

\section{M. abscessus phage tree}

The split network based on prophage gene content (i.e., the presence or absence of orthologous proteins) showed an organization that differed from that of the M. abscessus tree (Figure 4B). The organization of splits in the $M$. abscessus phage split network differs from $M$. abscessus split network. M. massiliense type strain clusters with M. abscessus, while $M$. abscessus $4 S$ strain clusters with 


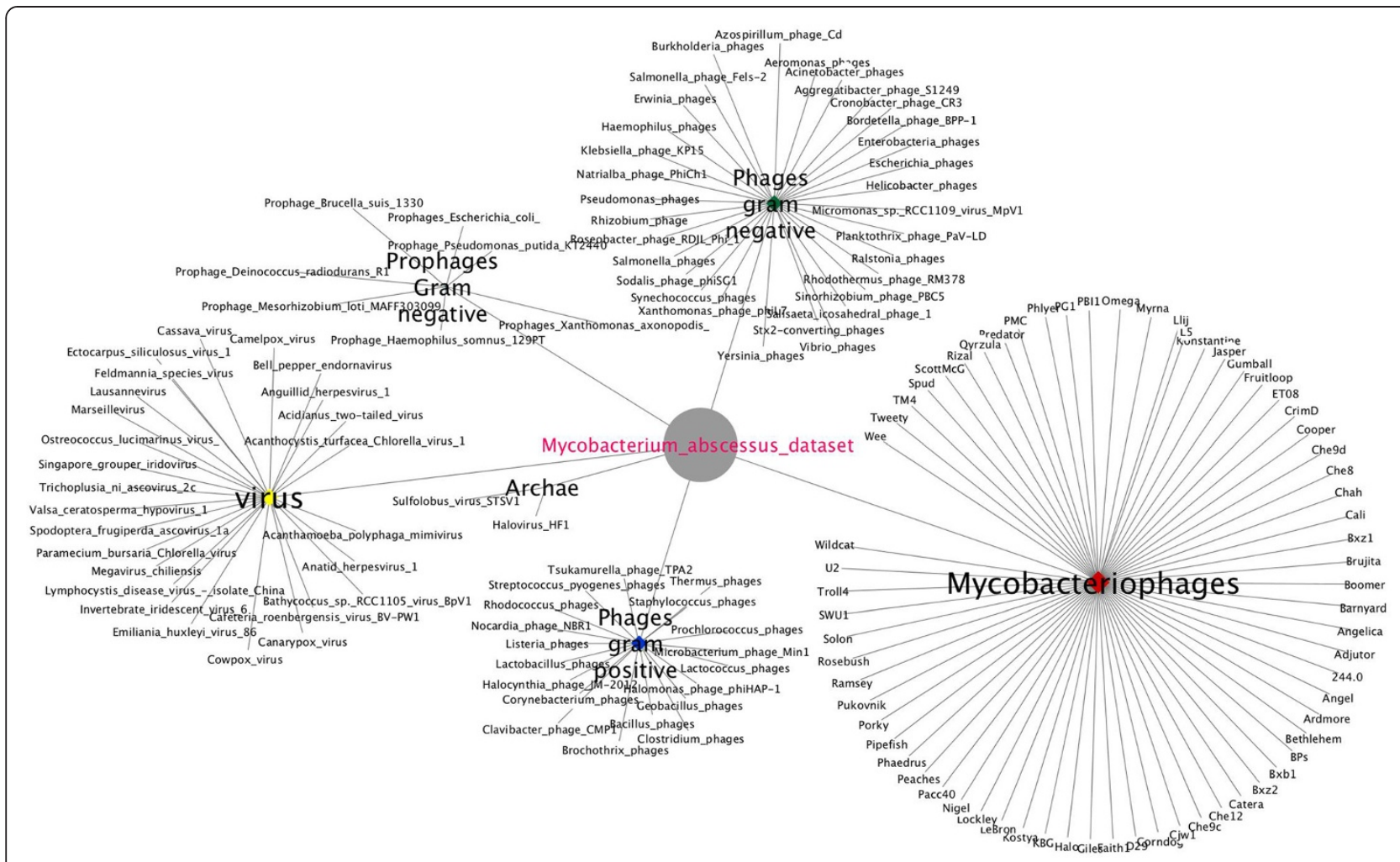

Figure $3 M$. abscessus viral and phage proteins annotation.

M. massiliense strains 2B and M159. The phage split network shows clusterization of $M$. massiliense 1S strains with $M$. bolletii while M. abscessus 6G strains forming a different cluster from $M$. abscessus. This phylogenomic analysis showed that $M$. abscessus viral and phage gene repertoires have different evolutionary histories. Also, a heatmap clusterization was constructed using a matrix of presence/absence of orthologous proteins. The heatmap clusterization showed a species organization different from that of the $M$. abscessus tree suggesting that $M$. abscessus may have been infected by several phages during their evolution (Figure 5). Likewise, using the tree based on whole-genome content, individual phylogenetic analysis for the different orthologous proteins groups revealed many topologies that differed from that of the M. abscessus tree. These results suggested that gene loss and HGT are relevant for all gene functions. Interestingly, Araucaria TMP clusters with M. massiliense strains $1 \mathrm{~S}$ and M172, suggesting that mycobacteriophages infecting $M$. massiliense mycobacteria may have features similar to Araucaria.

\section{Detection of HGT cases}

Among the 853 orthologous groups, phylogenetic trees were successfully reconstructed for $213(25 \%)$ of the cases, 156 transfer events (Additional file 3) were detected out of which 33 cases were associated with strong boostrap support for HGT. A $45.45 \%$ proportion of the transferred proteins are homologous to mycobacteriophages proteins, $12.12 \%$ homologous to viral proteins, $21.21 \%$ to proteins of phages infecting gram-positive bacteria and $21.21 \%$ to proteins of phages infecting gram-negative bacteria. The probable sources are environmental bacteria in 33 cases, M. abscessus in 13 cases, M. bolletii in 6 cases and M. massiliense in 4 cases.

\section{Discussion}

Analysing 171 prophage regions in $47 M$. abscessus complex genomes indicated that $M$. abscessus complex has been infected by at least 17 different mycobacteriophages, including Araucaria, the sole available mycobacteriophage that we recently isolated from $M$. bolletii [23]. Noteworthy, M. abscessus M154, an isolate from Malaysia [38] is the only strain lacking any evidence for phage. Our previous analysis revealed no unique genes in this strain [39], which nevertheless encodes four possible CRISPRs, one cmr1 family and one cmr4 family, with potential immunity against phage infection [40]. Alternatively, no phage was detected in this strain because of database limitations. For example, Araucaria did not yield significant homology with any other $M$. abscessus complex phage, as confirmed by phylogenetic studies using whole viral and phage proteins clusterization and TMP protein sequence based tree. Sequencing additional mycobacteriophages may next reveal prophage regions in $M$. abscessus M154. 
A

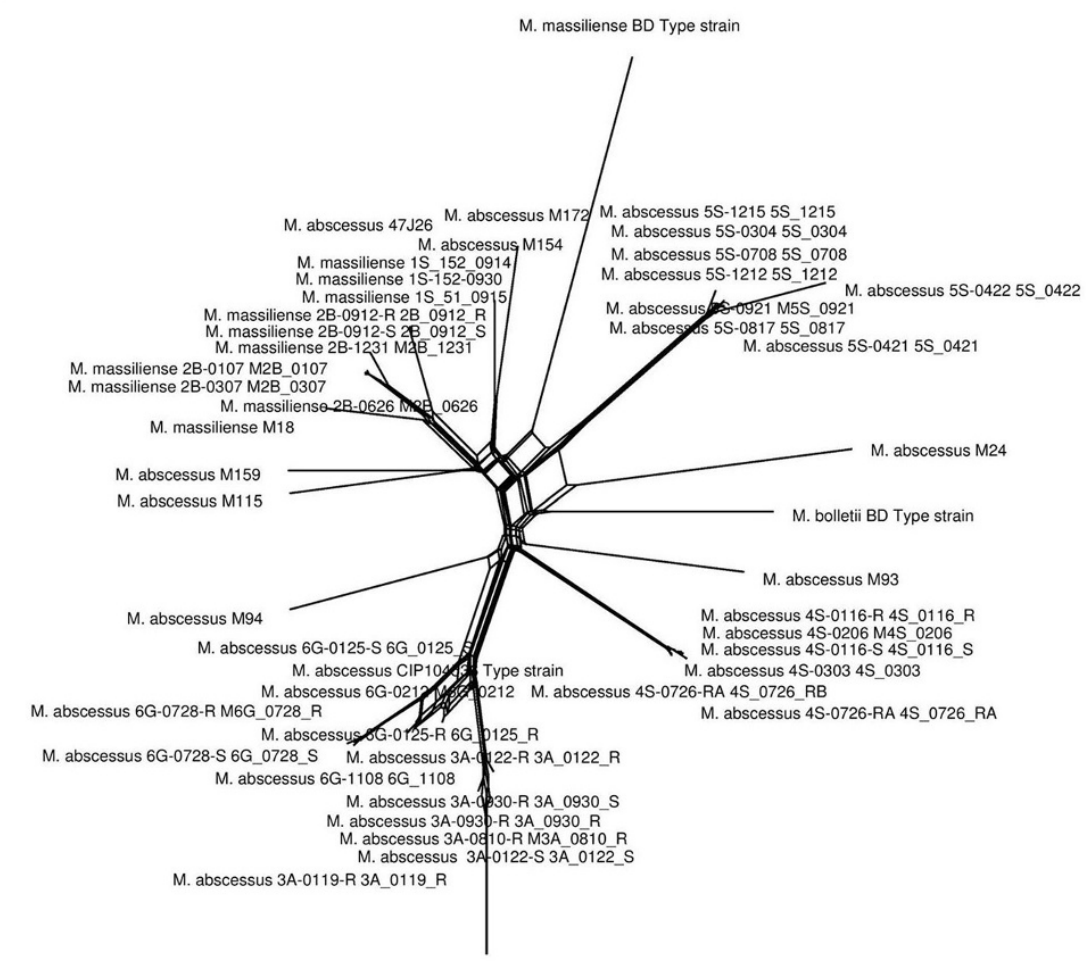

B

M. abscessus 3A-0731 3A_0731

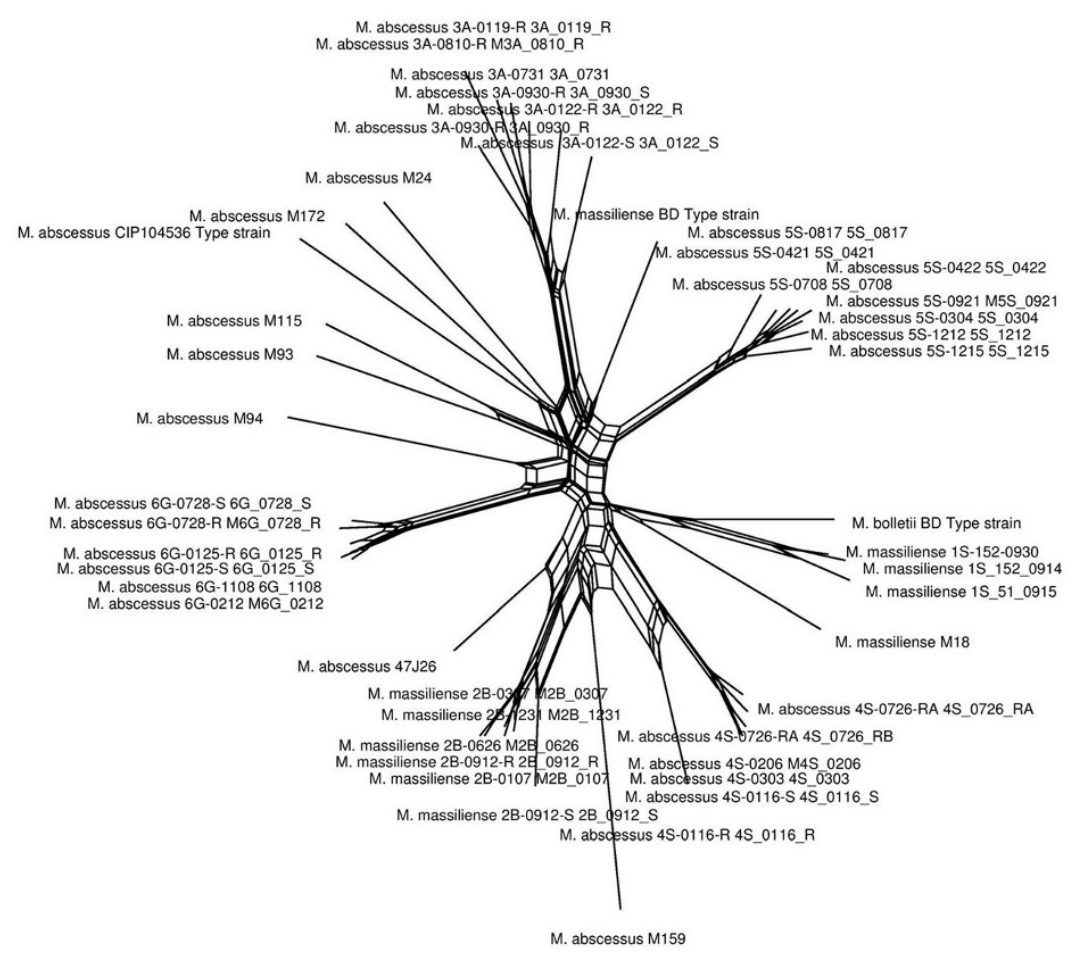

Figure 4 (See legend on next page.) 
(See figure on previous page.)

Figure 4 M. abscessus phylogeny. A- M. abscessus species split network. B- $M$. abscessus phage split network based on phage gene content matrix. Every edge is associated with a split of the taxa, but there may be a number of parallel edges associated with each split. The edges separate taxa on one side of the split from the taxa on the other side of the split. The length of an edge in the network is proportional to the weight of the associated split. This is analogous to the length of a branch in a phylogenetic tree.

We further observed that some M. abscessus phage proteins had homology to other mycobacteriophages and to phages infecting environmental bacteria. M. abscessus complex mycobacteria are opportunistic pathogens, but these observations suggest that environments, rather than host microbiota, are sources of evolution for M. abscessus complex mycobacteriophages. Noteworthy, potential sources are living in amoeba (Additional file 4) where M. abscessus complex mycobacteria including M. massiliense [22] and M. bolletii [41] are also residing. Amoeba are a place for DNA exchanges between sympatric organisms and the amoeba themselves [42-48]. Data here reported suggest that amoeba are a likely place for mycobacteriophage exchanges and therefore, may be a place to look at for the discovery of new mycobacteriophages.
Accordingly, a striking feature of $M$. abscessus complex phage genomes is their pervasive mosaicism, a previously reported hallmark of mycobacteriophages [49,50]. Our phylogenomic and phylogenetic analyses revealed a different split network topology between the hosts and the phages. This probably reflects reciprocal genome evolution through a dynamic co-evolutionary process [51]. M. abscessus complex was infected by at least 17 phages and these infections contributed to differentiate $M$. abscessus complex into several clusters of mycobacteria. Widespread occurrence of phage sequences in almost all studied $M$. abscessus complex isolates suggests that the rate of prophage invasion is faster than the rate of mutation, implying rapid evolution of $M$. abscessus. Also, in M. abscessus complex a total of 6/33 (19\%) gene transfers
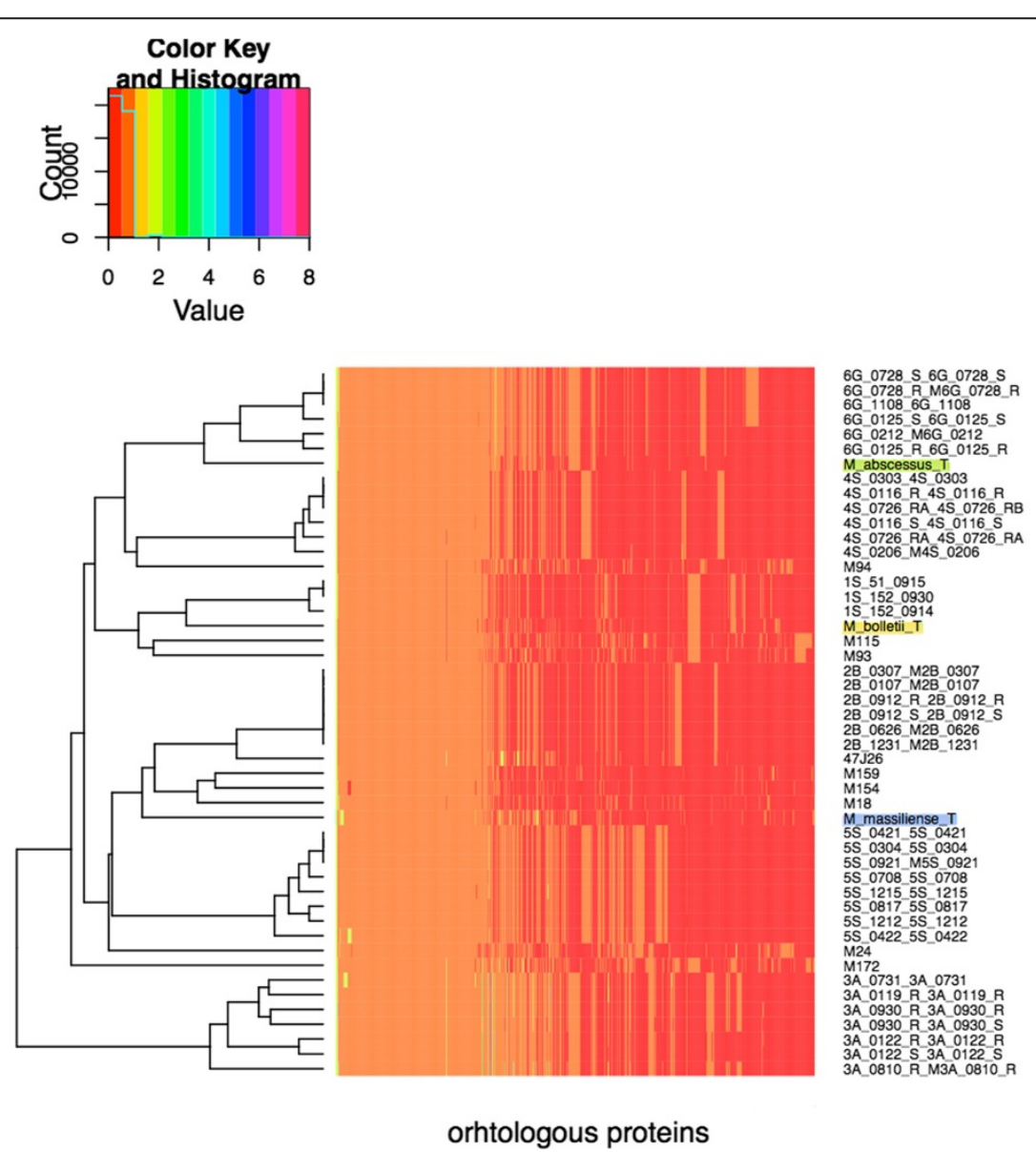

Figure 5 Heatmap clusterization based on protein presence absence matrix. 
occurred between a set of donor species (at least two donors) and a recipient species and 27/33 (81\%) gene transfers occurred between a single species donor and a recipient species, clearly indicating it is an on-going process.

\section{Conclusions}

Excluding the prophage-free strain M154, phages account for only $6.7-9.6 \%$ of $M$. abscessus complex genomic content, but they profoundly impact their hosts, participating to their on-going diversification.

\section{Reviewers' comments}

We appreciate the reviewer's comments from Dr. Eric Bapteste (UPMC, Institut de Biologie Paris Seine, France), Dr. Patrick Forterre (Institut de Génétique Microbiologie, 91405 Orsay Cedex, France Institut Pasteur) and Dr. Eugene Koonin (National Center for Biotechnology Information, National Library of Medicine, National Institutes of Health, Bethesda, MD, USA). We have revised the manuscript according to your comments and suggestions.

\section{Reviewer 1: Dr. Eric Bapteste (UPMC, Institut de Biologie Paris Seine, France)}

The general topic of this research and the reported findings are very relevant for Biology Direct, however the current version of this MS is certainly not yet ready for publication.

Even though I am willing to trust the authors about their main conclusions, I strongly recommend major revisions, because it is currently hardly possible to evaluate most of the evidence on which they based their observations.

In short:

- The trees supporting lateral gene transfers should be presented in an organized fashion in a Supp. Mat.

Authors' response: The reviewer is right, all 605 trees are now provided as Additional File and trees supporting LGT are highlighted.

- Many current figures are not of sufficient quality to be printed in a journal (gene/taxon names are impossible to read, etc.)

Authors' response: Authors improved the quality of documents.

- The methodology used for the tree reconstruction is not sufficiently detailed: crucial information such as the number of positions retained or the substitution models used are lacking.

Authors' response: Methodology has been expanded (Lines 87-92; lines 100-102; lines 112-122).

- Many figures presented in the text are underinterpreted, and not critically discussed.

Elements requiring significantly more details:

p.4. 1. 86: The authors report that 'M. abscessus proteomes were aligned using Mauve software [24]'. I am not familiar with ProgressiveMauve, but does this software really align proteomes, or is it rather a tool to align genomes based on their content and gene order? If so, the next sentence in the MS is hard to understand

Authors' response: $M$. abscessus genomes were aligned using Mauve software. Progressive Mauve uses Muscle or clustlW to perform alignment. Here we used Muscle. The authors corrected and explained the methodology (P.4 line 86).

'Then M. abscessus tree was constructed using Neighbor-Net algorithm in the package SplitsTree'. What distance matrix was provided to Splitstree? What was this distance reflecting?

Authors' response: Mauve alignment generates an identity matrix file which calculates the identity score range between 0 and 1; 0 indicates no homologous nucleotides and 1 indicates that every homologous nucleotide was identical. The authors explained this part in the text (P.5 line 88).

If the proteomes alignments evoked above were 'classic' protein alignments, then what happened to poorly aligned sites? How many positions were retained? For how many proteins? The material and methods must be much more detailed for the reader to really understand the analysis and the results. Please note that this criticism also applies for the trees that show some LGT.

Authors' response: The authors corrected that $M$. abscessus genomes was aligned not proteomes in the text (P.4 line 86).

p.5. 1, 95-96: The analysis described here faces a potential pitfall if 'presence' and 'absence' were treated in a symmetric fashion, especially if there were lots of 'absences' in this matrix. While 'presences' can be used to group genomes sharing some features, more caution is required in the use of shared 'absences'.

If the groupings of genomes are firstly caused by the lack of shared features, then these groupings can be very artefactual (sharing 'losses' is different from sharing nothing. The potential problem here is that two genomes that have positively nothing, or not much in common, may still be grouped due to their lack of genes, while sometimes it is a better idea not to group genomes that share nothing in common!). How did the authors address this potential issue?

Authors' response: Here we constructed a matrix based on the similarity between two species which is defined as the number of genes that they have in common divided by their total number of genes (P.5 line 100-104).

p.5, 1. 98-108: Incongruence between trees is considered to be synonym of lateral gene transfer, and the possibility of tree reconstruction artefacts is not discussed. Since the tree reconstruction methods are poorly described, it is difficult to evaluate this part of the work. 
Authors' response: The methods of tree reconstruction are now described in the materials and methods section. (P.5 line 105-124).

p.6., 1. 119: The authors write that 'a phylogenetic tree was constructed based on Tape Measure Proteins (TMP)' What are 'Tape Measure Proteins'? Why this marker? What methods/positions/models were used to reconstruct this tree?

Authors' response: The TMP was selected because it is typically the longest gene in mycobacteriophage genomes and because regions within TMP gene are conserved (P.6 line 125).

p.7, 1. 156. Figure 4A: I have not been able to see this figure, or if it refers to the split network, then its description in the text must be expanded and be more critical. What do the proportion/size/presence of splits indicate? What is their biological meaning? Also for example, what does the position of M. massiliense BD type strain suggest? Same question for the position of M. abscessus $47 \mathrm{~J} 26$ ?

Authors' response: The authors performed a better figure quality and more description in the text. The length of an edge in a split network is analogous to the length of a branch in a phylogenetic tree.

p.7, 1.160: same problem with Figure 4B.

Authors' response: The authors performed a better figure quality and more description in the text (P.7 lines 158-160).

Overall, the exploitation of these 2 figures is a bit vague. The authors only write about it that: 'A phylogenomic tree based on prophage gene content (i.e., the presence or absence of orthologous proteins) showed an organization that differed from that of the M. abscessus tree (Figure 4B).' Please, increase the descriptions of what these differences are (or use a metrics to compare these two split networks).

Authors' response: The authors performed a better figure quality and more description in the text (P.8 line 186-190).

p.8, 1.165: Figure 5 is neither described nor exploited in a way that allows to make sense of the main text about it. Please, give more time to a careful critical description of the figure.

Authors' response: The authors clarified this point (P.8 line 174).

p.8, 1. 172: The 214 phylogenetic trees mentioned here (reconstructed how, please precise) should be logically classified and presented as Supp. Mat, or made available somewhere. Currently, it is simply impossible to review this part of the MS without being able to look at the evidence.

Authors' response: The phylogenetic trees are provided in Additional file 4.

p.10. 1-210-211: 'Also, M. abscessus complex phages further shuttled gene transferts, 16/29 (55\%) of which occurred between different $M$. abscessus complex clusters but $13 / 29$ (45\%) of which occurred between strains of the same cluster, clearly indicating it is an on-going process'. Where do these numbers come from? How were they obtained? Where is the evidence?

Authors' response: The authors clarified this point in the text (P.10 line 241-246).

p.19, 1. 379: Figure 2 legend: What is 'an interacting map'? How is one supposed to read such a map? What are the nodes? What are the edges? More descriptions are required.

Authors' response: The authors clarified this point (P.19 line 422).

There are also some minor typos/issues:

p.3, 1. 56 'three different organisms': do you really mean organisms, or species, or strains here?

Authors' response: We mean organisms.

p.4, 1.86 (and in some other places in the text): the authors refer to the splitsnetwork as the ' $M$. abscessus tree'. Elswhere, as in the legend of Figure 4, they call this type of graphs 'network trees'. This wording is confusing. Is it a network or is it a tree? To me, each of this graph should be called a split network.

Authors' response: The authors corrected network tree to split network (P.5 line 91).

p.5., 1. 95: Likewise, what the authors call 'a wholegenome phylogenetic tree' looks very much like a network.

Authors' response: The authors corrected phylogenetic tree to network (P.5 line 100).

Figure 2: 'Few exceptions were observed: strains M139 and 1S_51_0915 showed prophage region homology with the M. abscessus cluster while M. bolletii M24 showed prophage region homology with the $M$. massiliense cluster'. Please help the reader more to see this, it is impossible to guess where the strains discussed here are in this map, add some arrows.

Figure 3 is likely too large in its current format for publication.

Authors' response: The authors improved the quality of Figures.

p.6, 1.136: 'Interestingly". Why? Please explain why it is interesting.

Authors' response: The authors clarified this point (P.7 line 137).

SI 1 \& 2: 'porphages' should be prophages Figure legends: Figure 1: 'Phylogentic' must be fixed + see problems with Figure 4 and Figure 2 legends discussed above.

Authors' response: The authors corrected this point.

p.34. Table three: what is the difference between a 'parent species' and a 'donor species?' ('donor' takes only 1 'n'). What does the column ' $\mathrm{Nb}$ Duplications before parent' refers to? 
Authors' response: The parent species is the node which contains the two sub-trees: recipient and donor species and the number of duplication before parent refers to the number of gene duplication before the HGT event.

\section{Second revision requested be the Reviewer 1: Dr. Eric Bapteste (UPMC, Institut de Biologie Paris Seine, France)}

The revised version of the MS by Sassi et al. is improved. I am still uncertain whether the quantification of HGT using gene trees means much biologically. I suppose this is because I doubt that trees alone convey that kind of evidence anyway. I am more convinced by studies of synteny showing prophages with similar genes inserted at various positions of Mycobacterium genomes. I remain also unconvinced (to be honest somewhat skeptical) about the quality of the figures. I suppose this latter possible issue would be addressed by the publisher then.

p.9. 1. 195. The content of Additional file 4 is useful, also not yet perfect for its purpose: both trees with and without candidate HGT are present in this file (i.e. there are around 214 trees in it, not 156 trees), making it difficult to evaluate the trees with HGT only. The legend for this file (p.27, 1.614) is confusing as it seems to announce 214 trees with candidate HGT. When one looks at some of these tree files however, one finds the following associated description: 'None horizontal gene transfert event'. (So some of these trees should be removed from Additional file 4, and the English of this final description could be improved).

Authors' response: In the additional file 4 we changed the report by trees as figures and it presents 75 trees representing a total of 156 transfer events. The HGT is represented by yellow squares in the figures. The legend is corrected accordingly to the reviewer's comment.

p.9. 1.199-201: When discussing the sources of HGT, I find it strange that the category 'unknown' is not quantified, nor discussed. In the few trees I have looked at from additional file 4, 'unknown' was the major HGT donor...

Authors' response: The category "unknown" in the report files represents the taxonomy used in the project which is not that same used by NCBI. As the species name is too long we removed it and only the strain name is presented, meaning that the program we run does not recognize the names we gave in data. Here we present as additional file 4 the figures of the trees to avoid any confusion.

p.10. 1. 227. 'a different topology', sure, but a topology of what? I suppose of split networks. Indeed, the networks look different, but note that their difference is still not assessed by any formal distance computed between them, nor by any statistical test. Some might find that this aspect of the study would have deserved to be improved.

Authors' response: Indeed a different split network topology. This is corrected in the text Page10, line 227.

All the minor comments were corrected.

\section{Reviewer 2: Dr. Patrick Forterre (Institut de Génétique Microbiologie, 91405 Orsay Cedex, France Institut Pasteur)}

The authors have analyzed the proviruses integrated in 48 strains of the Mycobacterium abscessus complex. Interestingly, this analysis allowed detecting six new families of mycobacterioviruses, in addition to the previously described virus Araucaria. The authors observe that these viruses roughly co-evolved with their hosts since they can be divided in three clusters corresponding to the three Mycobacterium abscessus sub-complexes. However, they also noticed many incongruence between various tree topologies that are interpreted as horizontal gene transfer (HGT). It is unclear for me which of these transfer correspond to independent gain and/or loss of proviruses in different lineages of $M$. absessus and which ones are due to real transfer of viruses from one lineage to the other. It is also not clear why the authors concluded that viral infection contributes to the differentiation of the M. abscessus complex.

The presentation of Figures and Table could be improved. The Tables 1 and 2 could be placed in supplementary material and important information about the proviruses summarized in Figure (diagram) and/or Table (how many genomes have $0,1,2 \ldots .7,8$ integrated elements, size distribution, main features of the four classes proposed). The trees/networks are also difficult to interpret.

There are several minor points Lane 123; define prophage region homology.

Authors' response: This methodology section has been re-written (Lines 112-123).

Lane 128: 242,067 proteins (which proteins?) in general be more precise

Authors' response: The authors clarified this point (P.7 line 139).

Lane 140 and elsewhere, gram-positive bacteria is no more a valid taxonomic grouping, better to indicate Firmicutes

Authors' response: The authors corrected this point (P.7 line 151).

Lane 168: Auracaria should be Araucaria

Authors' response: The authors corrected this point (P.8 line 182).

Lane 193: gram-negative and gram positive bacteria! i.e. all bacteria except mycobacteria?? So environmental bacteria is sufficient.

Authors' response: The authors corrected this point (P.9 line 206). 
Table 2 M. abscessus HGT cases

Putative HGT

Parent species

Recipient species

Donor species

$\mathrm{Nb}$ duplications

PHAGE_Mycoba_Peaches-gi|282598664|ref|YP_003358761.1/gp58

[M93]

[6G-0125-R]

[M93]

before parent

[Mycobacterium_phage_Peaches]

PHAGE_Plankt_PaV_LD-gi|371496158|ref|YP_004957306.1|ABCtransporter [PlanktothrixphagePaV-LD]

[PSEUDO - 5S-0421]

[M94]

[M93 M115]

4

PHAGE_Mycoba_LeBron-gi|304360967|ref|YP_003857149.1|gp18 [Mycobacterium_phage_LeBron]

[M115]

[M172]

[M115]

2

PHAGE_Mycoba_Giles-gil160700672|ref|YP 001552352.1|gp23 [Mycobacterium_phage_Giles]

PHAGE_Mycoba_Che9c-gi|29566118|ref|NP_817687.1|gp10 [Mycobacterium_phage_Che9c]

PHAGE_Mycoba_Pukovnik-gi|192824238|ref|YP_001994879.1|gp62 [Mycobacterium_phage_Pukovnik]

PHAGE_Tricho_2c-gi||1 16326757|ref|YP_803294.1/hypotheticalproteinTNAV2c_gp071 [Trichoplusia_ni_ascovirus_2c]

PHAGE_Salmon_PVP_SE1-gi|363539742|ref|YP_004894027.1|hypothetical protein [SalmonellaphagePVP-SE1]

PHAGE_Rhodoc_REQ3-gi|372449972|ref|YP_005087193.1|phageintegrase [RhodococcusphageREQ3]

PHAGE Salmon PVP SE1-gi|363539618|ref|YP 004893903.11 phosphoribosylpyrophosphatesynthetase[SalmonellaphagePVP-SE1]

PHAGE_Mycoba_Myrna-gi|203454746|ref|YP_002225062.1|gp183 [Mycobacterium_phage_Myrna]

PHAGE_Mycoba_Omega-gi|29566822|ref|NP_818386.1|gp85 [Mycobacterium_phage_Omega]

PHAGE_Mycoba_Pacc40-gi|206600097|ref|YP_002241602.1|gp18 [Mycobacterium_phage_Pacc40]

PHAGE_Mycoba_Pacc40-gi|206600097|ref|YP_002241602.1/gp18 [Mycobacterium_phage_Pacc40]

PHAGE_Acanth_mimivirus-gi|31 1977570|ref|YP_003986690.1|DNAtopoisomerase1b [Acanthamoebapolyphagamimivirus]

PHAGE_Rhodoc_RER2-gi|372449922|ref|YP 005087145.1/hypotheticalprotein

$$
\text { [RhodococcusphageRER2] }
$$

PHAGE_Aeromo_31-gi|66391812|ref|YP 238737.1/hypotheticalproteinPHG31p8 [Aeromonas_phage_31]

PHAGE_Lactoc_P087-gi|229605000|ref|YP_002875699.1|putativecysteinesynthase [Lactococcus_phage_P087]

$\begin{array}{lccc}{[\text { M94 }]} & {[6 \mathrm{G}-0728-\mathrm{R}]} & {[\mathrm{M} 94]} & 5 \\ {[\mathrm{M} 18]} & {\left[3 \mathrm{~A}-0122 \_S\right]} & {[\mathrm{M} 18]} & 2 \\ {[\mathrm{M} 154]} & {[\mathrm{M} 115]} & {[\mathrm{M} 154]} & 5\end{array}$

[5S-0921]

[M. massiliense T]

$$
\text { [M172] }
$$

[3A-0122_S1]

[M172]

[M115]

[M24]

[PSEUDO - M159]

[3A-0122_S7]

[M24]

[4S-0726]

[47 J26]

[M172]
[4S-0726]

[5S-0921]

[M. massiliense T]

[M172]

[3A_0930_S]

[M. massiliense T]

[3A-0122_S1]

[M172]

[M115]

[M24]

[M172 3A-0122_S6 3A-0122_S2 3A-0122_S4 47 J26]

[3A-0122_S7]

[M24]

[4S-0726]

[47 J26]

[M172] 
Table 2 M. abscessus HGT cases (Continued)

20 PHAGE_Plankt_PaV_LD-gi|371496158|ref|YP_004957306.1|ABCtransporter

$$
\text { [PlanktothrixphagePaV-LD] }
$$

21

PHAGE_Acanth_mimivirus-gi|311977513|ref|YP_003986633.1| putativedTDP-D-glucose4,6-dehydratase[Acanthamoebapolyphagamimivirus]

PHAGE_Bacill_36-gi|156564011|ref|YP_001429750.1|PcrAhelicase [Bacillus_phage_0305phi8_36]

PHAGE_Mycoba_Che9c-gi|29566174|ref|NP_817745.1|gp68[Mycobacterium_phage_Che9c] PHAGE_Mycoba_Che8-gi|29565783|ref|NP_817355.1|gp17[Mycobacterium_phage_Che8]

PHAGE_Tricho_2c-gi||116326757|ref|YP_803294.1|hypotheticalproteinTNAV2c_gp071

$$
\text { [Trichoplusia_ni_ascovirus_2c] }
$$

PHAGE_Microm_MpV1-gi|313768434|ref|YP_004062114.1|hypotheticalprotein [Micromonassp.RCC1109virusMpV1]

PHAGE_Mycoba_Pipefish-gi|109521870|ref|YP_655307.1|gp30

[Mycobacterium_phage_Pipefish]

$$
\begin{gathered}
\text { PHAGE_Plankt_PaV_LD-gi|371496158|ref|YP_004957306.1|ABCtransporter } \\
\text { [PlanktothrixphagePaV-LD] }
\end{gathered}
$$

PHAGE_Lactoc_P087-gi|229605000|ref|YP_002875699.1|putativecysteinesynthase

$$
\text { [Lactococcus_phage_P087] }
$$

PHAGE_Mycoba_Omega-gi|29566768|ref|NP_818332.1/gp31

$$
\text { [Mycobacterium_phage_Omega] }
$$

PHAGE_Burkho_phi1026b-gi|38707948|ref|NP_945089.1|gp58

$$
\text { [Burkholderia_phage_phi1026b] }
$$

PHAGE_Mycoba_Cjw1-gi|29565933|ref|NP_817504.1|gp55[Mycobacterium_phage_Cjw1]
[3A-0122_S5]

[3A-0122_S4]

[3A-0122_S7]

[4S-0726-RA]

[3A-0119-R]

[M18]

[M24]

[M115]

[M115]

[M18]

[M. bolletii T]

[M24]

[M94]

[PSEUDO -M154]

[PSEUDO -M159]

$$
\begin{gathered}
\text { [M94] } \\
\text { [M172 3A-0122_S4 } \\
\text { 3A-0122_S2 47 J26] }
\end{gathered}
$$

[4S-0116_S]

[M94]

[M172]

[4S-0726-RA]

[M. bolletii T]

[6G-1108]

[M. bolletii T]

[M. bolletii T] TM154 M172 M159]

[M172 3A-0122_SO

1S-152-0930

3A-0122 S1

[M115]

[M24]

[M115] 


\section{Reviewer 3: Dr. Eugene Koonin (National Center for Biotechnology Information, National Library of Medicine, National Institutes of Health, Bethesda, MD, USA)}

The importance of bacteriophage contribution to the evolution of bacterial genomes is increasingly recognized. Here Sassi and coworkers conclude that bacteriophages drive the evolution of the Mycobacterium abscessus complex. I find this appealing and credible idea but fail to see how the data presented in the manuscript, even assuming that the identification of prophages is accurate (no specific evidence of that is provided), support such a strong conclusion. I can agree that the authors demonstrate differences in the prophage content between the bacteria in the complex. Then, I suppose, the argument would be that the trees of the bacteria and phages are different, suggesting that there has been some exchange of prophages and individual genes. The robustness of the trees is a concern because the trees for phages can be notoriously difficult. But, even assuming they are correct, this argument seems to fall far short of the ambitious claim of the paper. It could be very helpful if the authors made an effort to carefully present their logic.

Authors' response: The authors revised the manuscript in light of the reviewer comments to further discuss the impact of mycobacteriophages on the on-going diversification of this group of mycobacteria. (Lines 245-250).

\section{Additional files}

Additional file 1: Mycobacterium abscessus genome properties and their prophage regions.

Additional file 2: Mycobacterium abscessus CRISPRs and the correlation with number of prophage regions.

Additional file 3: The reconstructed trees for HGT events. Each tree contains one to six HGT events. The yellow squares represent the HGT event

Additional file 4: Environmental bacteria hosting homologous M. abscessus phage proteins and evidence for bacteria-amoeba interaction.

Competing interests

The authors declare that they have no competing interests.

\section{Authors' contributions}

MS, PG and OC performed the analyses. MS and MD designed the study. MS, PP and MD interpreted data and wrote the draft. All authors read and approved the final manuscript.

\section{Acknowledgments}

MS was financially supported by Infectiopole Sud Foundation.

\section{Author details}

${ }^{1}$ Unité de Recherche sur les Maladies Infectieuses et Tropicales Emergentes UMR CNRS 6236 IRD198, IFR48, Institut Méditerranée Infection, Aix Marseille Université, Marseille, France. ${ }^{2}$ I2M, UMR 7373, EBM 13331Aix Marseille Université, CNRS, Centrale Marseille, Marseille, France.

Received: 5 March 2014 Accepted: 7 August 2014

Published: 15 September 2014

\section{References}

1. Ripoll F, Pasek S, Schenowitz C, Dossat C, Barbe V, Rottman M, Macheras E, Heym B, Herrmann JL, Daffé M, Brosch R, Risler JL, Gaillard JL: Non mycobacterial virulence genes in the genome of the emerging pathogen Mycobacterium abscessus. PLoS One 2009, 4:e5660.

2. Furuya EY, Paez A, Srinivasan A, Cooksey R, Augenbraun M, Baron M, Brudney K, Della-Latta P, Estivariz C, Fischer S, Flood M, Kellner P, Roman C, Yakrus M, Weiss D, Granowitz EV: Outbreak of Mycobacterium abscessus wound infections among "lipotourists" from the United States who underwent abdominoplasty in the Dominican Republic. Clin Infect Dis 2008, 46:1181-1188

3. Viana-Niero C, Lima KV, Lopes ML, Rabello MC, Marsola LR, Brilhante VC, Durham AM, Leão SC: Molecular characterization of Mycobacterium massiliense and Mycobacterium bolletii in isolates collected from outbreaks of infections after laparoscopic surgeries and cosmetic procedures. J Clin Microbiol 2008, 46:850-855.

4. Koh WJ, Jeon K, Lee NY, Kim BJ, Kook YH, Lee SH, Park YK, Kim CK, Shin SJ, Huitt GA, Daley CL, Kwon OJ: Clinical significance of differentiation of Mycobacterium massiliense from Mycobacterium abscessus. Am J Respir Crit Care Med 2011, 183:405-410.

5. Petrini B: Mycobacterium abscessus: an emerging rapid-growing potential pathogen. APMIS 2006, 114:319-328.

6. Hayes D Jr: Mycobacterium abscessus and other nontuberculous mycobacteria: evolving respiratory pathogens in cystic fibrosis: a case report and review. South Med J 2005, 98:657-661.

7. Griffith DE, Girard WM, Wallace RJ Jr: Clinical features of pulmonary disease caused by rapidly growing mycobacteria. An analysis of 154 patients. Am Rev Respir Dis 1993, 147:1271-1278.

8. Griffith DE: Emergence of nontuberculous mycobacteria as pathogens in cystic fibrosis. Am J Respir Crit Care Med 2003, 167:810-812

9. Olivier KN, Weber DJ, Wallace RJ Jr, Faiz AR, Lee JH, Zhang Y, Brown-Elliot BA, Handler A, Wilson RW, Schechter MS, Edwards LJ, Chakraborti S, Knowles MR: Nontuberculous mycobacteria. I: multicenter prevalence study in cystic fibrosis. Am J Respir Crit Care Med 2003, 167:828-834.

10. Pierre-Audigier C, Ferroni A, Sermet-Gaudelus I, Le Bourgeois M, Offredo C, Vu-Thien $H$, Fauroux B, Mariani P, Munck A, Bingen E, Guillemot D, Quesne G, Vincent V, Berche P, Gaillard JL: Age-related prevalence and distribution of nontuberculous mycobacterial species among patients with cystic fibrosis. J Clin Microbiol 2005, 43:3467-3470.

11. Chalermskulrat W, Sood N, Neuringer IP, Hecker TM, Chang L, Rivera MP, Paradowski LJ, Aris RM: Non-tuberculous mycobacteria in end stage cystic fibrosis: implications for lung transplantation. Thorax 2006, 61:507-513.

12. Jonsson BE, Gilljam M, Lindblad A, Ridell M, Wold AE, Welinder-Olsson C: Molecular epidemiology of Mycobacterium abscessus, with focus on cystic fibrosis. J Clin Microbiol 2007, 45:1497-1504.

13. Levy I, Grisaru-Soen G, Lerner-Geva L, Kerem E, Blau H, Bentur L, Aviram M, Rivlin J, Picard E, Lavy A, Yahav Y, Rahav G: Multicenter cross-sectional study of nontuberculous mycobacterial infections among cystic fibrosis patients, Israel. Emerg Infect Dis 2008, 14:378-384.

14. Griffith DE, Aksamit T, Brown-Elliott BA, Catanzaro A, Daley C, Gordin F, Holland SM, Horsburgh R, Huitt G, lademarco MF, Iseman M, Olivier K, Ruoss S, von Reyn CF, Wallace RJ Jr, Winthrop K, ATS Mycobacterial Diseases Subcommittee, American Thoracic Society, Infectious Disease Society of America: An official ATS/IDSA statement: diagnosis, treatment, and prevention of nontuberculous mycobacterial diseases. Am J Respir Crit Care Med 2007, 175:367-416.

15. Macheras E, Roux AL, Bastian S, Leão SC, Palaci M, Sivadon-Tardy V, Gutierrez C, Richter E, Rüsch-Gerdes S, Pfyffer G, Bodmer T, Cambau E, Gaillard JL, Heym B: Multilocus sequence analysis and rpoB sequencing of Mycobacterium abscessus (sensu lato) strains. J Clin Microbiol 2011, 49:491-499.

16. Sassi M, Ben Kahla I, Drancourt M: Mycobacterium abscessus multispacer sequence typing. BMC Microbiol 2013, 13:3.

17. Bryant JM, Grogono DM, Greaves D, Foweraker J, Roddick I, Inns T, Reacher M, Haworth CS, Curran MD, Harris SR, Peacock SJ, Parkhill J, Floto RA: Whole-genome sequencing to identify transmission of Mycobacterium abscessus between patients with cystic fibrosis: a retrospective cohort study. Lancet 2013, 381:1551-1560.

18. Leao SC, Tortoli E, Viana-Niero C, Ueki SY, Lima KV, Lopes ML, Yubero J, Menendez MC, Garcia MJ: Characterization of mycobacteria from a major Brazilian outbreak suggests that revision of the taxonomic status of 
members of the Mycobacterium chelonae-M. abscessus group is needed. J Clin Microbiol 2009, 47:2691-2698.

19. Leao SC, Tortoli E, Euzéby JP, Garcia MJ: Proposal that Mycobacterium massiliense and Mycobacterium bolletii be united and reclassified as Mycobacterium abscessus subsp. bolletii comb. nov., designation of Mycobacterium abscessus subsp. abscessus subsp. nov. and emended description of Mycobacterium abscessus. Int I Syst Evol Microbiol 2011, 61:2311-2313.

20. Cho YJ, Yi H, Chun J, Cho SN, Daley CL, Koh WJ, Jae Shin S: The genome sequence of 'Mycobacterium massiliense' strain CIP 108297 suggests the independent taxonomic status of the Mycobacterium abscessus complex at the subspecies level. PLoS One 2013, 8:e81560.

21. Adekambi T, Berger P, Raoult D, Drancourt M: rpoB gene sequence- based characterization of emerging non-tuberculous mycobacteria with descriptions of Mycobacterium bolletii sp. nov., Mycobacterium phocaicum sp. nov. and Mycobacterium aubagnense sp. nov. Int I Syst Evol Microbiol 2006, 56:133-143.

22. Adekambi T, Reynaud-Gaubert M, Greub G, Gevaudan MJ, La Scola B, Raoult D, Drancourt M: Amoebal coculture of "Mycobacterium massiliense" sp. nov. from the sputum of a patient with hemoptoic pneumonia. J Clin Microbiol 2004, 42:5493-5501.

23. Sassi M, Bebeacua C, Drancourt M, Cambillau C: The First Structure of a Mycobacteriophage, the Mycobacterium abscessus subsp. bolletii Phage Araucaria. J Virol 2013, 87:8099-8109.

24. Darling AE, Mau B, Perna NT: ProgressiveMauve: multiple genome alignment with gene gain, loss and rearrangement. PLoS One 2010, 5:e11147.

25. Zhou Y, Liang Y, Lynch KH, Dennis JJ, Wishart DS: PHAST: a fast phage search tool. Nucleic Acids Res 2011, 39:W347-W352. Web Server issue.

26. Hyatt D, Chen GL, Locascio PF, Land ML, Larimer FW, Hauser L: Prodigal: prokaryotic gene recognition and translation initiation site identification. BMC Bioinformatics 2010, 11:119.

27. Grissa I, Vergnaud G, Pourcel C: CRISPRFinder: a web tool to identify clustered regularly interspaced short palindromic repeats. Nucleic Acids Res 2007, 35:W52-W57. Web Server issue).

28. Huson DH, Bryant D: Application of Phylogenetic Networks in Evolutionary Studies. Mol Biol Evol 2006, 23:254-267.

29. Li L, Stoeckert J, Christian J, Roos DS: OrthoMCL: identification of ortholog groups for eukaryotic genomes. Genome Res 2003, 13:2178-2189.

30. Altschul SF, Madden TL, Schäffer AA, Zhang J, Zhang Z, Miller W, Lipman DJ: Gapped BLAST and PSI-BLAST: a new generation of protein database search programs. Nucleic Acids Res 1997, 25:3389-3402

31. Van Dongen S: Graph clustering by flow simulation. The Netherlands: PhD thesis. University of Utrecht; 2000 .

32. Snel B, Bork P, Huynen MA: Genome phylogeny based on gene content. Nat Genet 1999, 1:108-111.

33. Georgiades K, Merhej V, El Karkouri K, Raoult D, Pontarotti P: Gene gain and loss events in Rickettsia and Orientia species. Biol Direct 2011, 6:6.

34. Gouret P, Thompson JD, Pontarotti P: PhyloPattern: regular expressions to identify complex patterns in phylogenetic trees. BMC Bioinformatics 2009, 10:298.

35. Le PT, Ramulu HG, Guijarro L, Paganini J, Gouret P, Chabrol O, Raoult D, Pontarotti P: An automated approach for the identification of horizontal gene transfers from complete genomes reveals the rhizome of Rickettsiales. BMC Evol Biol 2012, 12:243.

36. Gouret P, Vitiello V, Balandraud N, Gilles A, Pontarotti P, Danchin EG: FIGENIX: intelligent automation of genomic annotation: expertise integration in a new software platform. BMC Bioinformatics 2005, 6:198.

37. Smith KC, Castro-Nallar E, Fisher JN, Breakwell DP, Grose JH, Burnett SH: Phage cluster relationships identified through single gene analysis. BMC Genomics 2013, 14:410.

38. Choo SW, Wong YL, Tan JL, Ong CS, Wong GJ, Ng KP, Ngeow YF: Annotated genome sequence of Mycobacterium massiliense strain M154, belonging to the recently created taxon Mycobacterium abscessus subsp. bolletii comb. nov. J Bacteriol 2012, 194:4778.

39. Sassi M, Drancourt M: Genome analysis reveals three genomospecies in Mycobacterium abscessus. BMC Genomics 2014, 15:359.

40. Koonin EV, Makarova KS: CRISPR-Cas: evolution of an RNA-based adaptive immunity system in prokaryotes. RNA Biol 2013, 10:679-686.
41. Adékambi T, Ben Salah S, Khlif M, Raoult D, Drancourt M: Survival of environmental mycobacteria in Acanthamoeba polyphaga. Appl Environ Microbiol 2006, 72:5974-5981.

42. Boyer M, Yutin N, Pagnier I, Barrassi L, Fournous G, Espinosa L, Robert C Azza S, Sun S, Rossmann MG, Suzan-Monti M, La Scola B, Koonin EV, Raoult D: Giant Marseillevirus highlights the role of amoebae as a melting pot in emergence of chimeric microorganisms. Proc Natl Acad Sci U S A 2009, 106:21848-21853

43. Penz T, Horn M, Schmitz-Esser S: The genome of the amoeba symbiont "Candidatus Amoebophilus asiaticus" encodes an afp-like prophage possibly used for protein secretion. Virulence 2010, 1:541-545.

44. Filée J, Chandler M: Gene exchange and the origin of giant viruses. Intervirology 2010, 53:354-361.

45. Colson P, Raoult D: Gene repertoire of amoeba-associated giant viruses. Intervirology 2010, 53:330-343.

46. Thomas V, Greub G: Amoeba/amoebal symbiont genetic transfers: lessons from giant virus neighbours. Intervirology 2010, 53:254-267.

47. Bertelli C, Greub G: Lateral gene exchanges shape the genomes of amoeba-resisting microorganisms. Front Cell Infect Microbiol 2012, 2:110.

48. Slimani M, Pagnier I, Raoult D, La Scola B: Amoebae as battlefields for bacteria, giant viruses, and virophages. J Virol 2013, 87:4783-4785.

49. Hatfull GF, Hendrix RW: Bacteriophages and their genomes. Curr Opin Virol 2011, 1:298-303.

50. Hatfull GF: The secret lives of mycobacteriophages. Adv Virus Res 2012, 82:179-288

51. Lindell D, Jaffe JD, Coleman ML, Futschik ME, Axmann IM, Rector T, Kettler G, Sullivan MB, Steen R, Hess WR, Church GM, Chisholm SW: Genome-wide expression dynamics of a marine virus and host reveal features of co-evolution. Nature 2007, 449:83-86.

doi:10.1186/1745-6150-9-19

Cite this article as: Sassi et al:: Mycobacteriophage-drived diversification of Mycobacterium abscessus. Biology Direct 2014 9:19.

\section{Submit your next manuscript to BioMed Central and take full advantage of:}

- Convenient online submission

- Thorough peer review

- No space constraints or color figure charges

- Immediate publication on acceptance

- Inclusion in PubMed, CAS, Scopus and Google Scholar

- Research which is freely available for redistribution 